\title{
$\begin{array}{ll}\text { Research Square They should not be considered conclusive, used to inform clinical practice, } & \text { Theprints are preliminary reports that have not undergone peer review. }\end{array}$ or referenced by the media as validated information. \\ Experimental Study On Antibacterial Properties of lodine-Coated Implant in Acute Osteomyelitis Model
}

\section{Xiqi Zhuang}

Fuzong Clinical Medical College of Fujian Medical University ${ }^{\circ}$ CFuzhou

Weibing Yang

Fuzong Clinical Medical College of Fujian Medical University ${ }^{\circ} \mathrm{CFuzhou}$

\section{Yongquan Zhang}

Fuzong Clinical Medical College of Fujian Medical University ${ }^{\circ}$ CFuzhou

Wanming Wang ( 914417805@qq.com)

Logistic Support Forces of The Chinese People's Liberation Army 900th Hospital

\section{Research Article}

Keywords: lodine-coated implant, Implant related infection, E. coli, lodine coating

Posted Date: May 10th, 2021

DOI: https://doi.org/10.21203/rs.3.rs-479954/v1

License: (c) (1) This work is licensed under a Creative Commons Attribution 4.0 International License. Read Full License 


\section{Abstract}

Purpose We have developed a iodine-coated implant and evaluated its antibacterial properties against Gramnegative bacteria by constructing an experimental osteomyelitis model.

Methods In this study, 16 titanium Kirschner-wires were selected, of which 8 titanium K-wires were treated with iodine on the surface by electrophoretic deposition with PVP-I solution. In our study, the standard strain of Escherichia coli (ATCC 25922) was selected, and 16 New Zealand rabbits were selected. There were 8 rabbits in the iodine-coated group and 8 rabbits in the non-iodine-coated group. All animals were drilled in the left proximal tibia after successful anesthesia.After that,the iodine-coated group was implanted with iodine-coated titanium Kwires. Then, $25 \mathrm{ul}$ of $2 \times 10^{8} \mathrm{CFU} / \mathrm{ml}$ bacterial suspension was injected into the bone marrow cavity with pipette gun, and the bone hole was sealed with bone wax.They were reared in cages for 1 week after operation, and evaluated and analyzed by microbiology, histopathology, scanning electron microscopy, etc.

Results The results of gross wound score and microbiology showed that the wound infection of the iodinecoated group was less severe than that of the non-iodine-coated group, which was confirmed by the histopathological results. Scanning electron microscopy and confocal microscopy showed that the amount of bacteria on the surface of iodine-coated K-wires was significantly lower than that non-iodine-coated K-wires.

Conclusions In this study, we have verified that the iodine-coated titanium implant could effectively inhibit E. coli infection in the early stage of infection by constructing an acute osteomyelitis model.

\section{Introduction}

Within become an important part of modern medicine, the implants have significantly improving the quality of life for the elderly ${ }^{1}$. However, the incidence of implant related infection (IRI) is also increasing. Studies have reported that the infection rate of hip and knee prosthesis is $1.9 \%$, while the infection rate of open fracture fixation is as high as more than $60 \%{ }^{2,3}$. Staphylococcus aureus bacteremia is the primary cause of internal plant infections, but the study of Bouvet et al. Show that the proportion of Gram-negative bacilli infection is higher than that of Gram-positive bacilli 2 years after implant ${ }^{4,5}$. Moreover, Escherichia coli is the primary cause of gram-negative bacteria IRI ${ }^{5,6}$. The pathogenesis of IRI is complex and the treatment is extremely difficult. The effect of traditional antibiotic treatment is limited ${ }^{7}$.

The current research focus is to improve the antibacterial properties of implant materials to achieve the purpose of preventing infection. At present, antibacterial coatings include antibiotic coatings,non-antibiotic organic antibacterial agent coatings, metal ion coatings, polyethyl- ene glycol coatings, surface modification, antimicrobial peptides and Chitosan and its derivatives ${ }^{8-14}$. Gentamicin coatings are the most widely used antibiotic coating in clinical practice. Studies have found that gentamicin coatings have greater advantages in the treatment of chronic osteomyelitis and prevention of postoperative infections ${ }^{8}$. However, the selection of sensitive antibiotics, the problem of bacterial resistance and the stable release of antibiotics in the coating and the maintenance of an effective bactericidal concentration are also important ${ }^{15,16}$.

At present, the concept of preventing implant infections emphasizes the surface modification of implants ${ }^{17}$. Different from Shirai et al. ${ }^{18-21}$, we have developed an iodine-coated titanium implant by electrophoretic 
deposition method 22,23 , which was verified to have clear antibacterial properties against Staphylococcus aureus by in experiments. However, at present, neither of the two iodine-coated titanium implants has carried out in vivo antibacterial experiments against Gram-negative bacteria.Gram-negative bacteria infections are also common in clinical orthopedics ${ }^{4-6}$.In this study, we will conduct an in vivo study on the antibacterial activity of titaniumcoated implants against Gram-negative bacteria by constructing an acute osteomyelitis model.

\section{Materials And Methods}

\section{Bacterial and Implant preparation}

A standard strain used in this experiment was Gram-negative Escherichia coli (ATCC 25922)(Department of Microbiology Laboratory).The inocula were prepared by subculturing the bacteria in $10 \mathrm{ml}$ of nutrient broth overnight at $37^{\circ} \mathrm{C}$ the day before inoculation. Each broth culture was then diluted in sterile PBS. The density of the inoculum was $2 \times 10^{8} \mathrm{CFU} / \mathrm{ml}$ of saline. In this experiment, 16 titanium K-wires with a length of $12 \mathrm{~mm}$ and a diameter of $1 \mathrm{~mm}$ were used to carry iodine on the surface in the laboratory of College of Materials and Engineering, Fuzhou University ${ }^{22}$.

\section{Animal model}

The experimental model followed a model in a previous study ${ }^{24}$ with slight modifications. Sixteen New Zealand rabbits with a body weight of $2.5 \pm 0.2 \mathrm{~kg}$ were selected in this experiment, are not limited to males and females.We determined the total number of experimental rabbits and the number of rabbits in each group according to the "resource equation" method.During the experiment,rabbits that died of anesthesia or infection less than 7 days were not included in the final statistics.All rabbits were caged preoperatively and fed adaptively for 7 days. They were randomly divided into iodine-coated group (numbered E1-E8) and non-iodine-coated group (numbered C1-C8), with 8 rabbits in each group by simple random classification.In this experiment, the blind method was used only after the experiment implementation process and during the outcome assessment. After the animals were treated, neither the experimenter nor the caregiver knew about the rabbit allocation team. Only assist personnel to know laboratory animal assignment team.All animals were anesthetized with $30 \mathrm{mg} / \mathrm{kg}$ sodium pentobarbital (1\%) via an auricular vein. The left hind leg of each rabbit was shaved from the knee to the ankle, and a simple tourniquet made of rubber band was tied above the left hind knee,cleaned with povidoneiodine, and draped with sterile sheets. Through the approach to the medial side of the left tibia, the bone marrow cavity was drilled with an electric drill with a $2.5 \mathrm{~mm}$ drill bit. And use a $2 \mathrm{ml}$ syringe needle to withdraw $0.2 \mathrm{ml}$ of bone marrow. The iodine-coated group was implanted with iodine-coated titanium K-wires, and the iodine-coated group was implanted with non-iodine-coated titanium K-wires. $25 \mathrm{ul}$ of $2 \times 10^{8} \mathrm{CFU} / \mathrm{ml}$ bacterial suspension was injected into the bone marrow cavity and the bone wax was used to seal the bone pores. Finally, the skin was closed using 4-0 silk threads(as shown in Fig. 1). After one week, all rabbits were observed on the injured limbs. The rabbits were killed by euthanasia and samples were collected for examination. The animal experiments were carried out in accordance with the ARRIVE guidelines. All methods were carried out in accordance with relevant guidelines and regulations. The study protocol was approved by the Experimental Animal Ethics Committee of 900th Hospital of PLA.

\section{Macroscopic score of wounds}


Observe and record the wounds of the affected limbs of rabbits. Using the method of Tang $\mathrm{H}$ et al. ${ }^{25}$, the gross observation score of the wounds was completed double-blindly.

\section{Microbiological evaluation}

Collect soft tissues and purulent secretions (if any) around K-wires in the medullary cavity.Place the specimen in a sterile test tube for examination. Then,they were incubated on blood agar for at least $24 \mathrm{~h}$ at $37^{\circ} \mathrm{C}$. The strain was analyzed by automatic bacterial identification system.

\section{Histopathologic evaluation}

The infected tibial tissue specimens were removed and fixed with $4 \%$ paraformaldehyde solution for $72 \mathrm{~h}$. All bone tissue specimens were decalcified with $5 \%$ formic acid decalcified solution for paraffin embedding, then they were stained with hematoxylin and eosin $(\mathrm{HE})$. A pathologist assisted in examining and scoring all sections under a conventional light microscope. The criteria for the diagnosis of osteomyelitis refer to the Jupiter scoring system ${ }^{26}$.By using the criteria described by Smeltzer et al. ${ }^{27,28}$, a numerical score was assigned to each variable, and all scores were added to create a composite histopathologic score with a maximum of 16, representing histopathologic severity.

\section{Scanning electron microscopy (SEM) and Confocal Laser Scanning Microscope(CLSM)}

The titanium K-wires were removed from the medullary cavity and cut into two equal lengths. Put one of the sections into a sterile culture cup containing $2.5 \%$ glutaraldehyde and fix it for 2 hours. After dehydration and drying, it is placed in an ion sputtering apparatus to spray gold.Then They were observed under SEM. The other section was stored in a $2.5 \%$ glutaraldehyde solution, and then two dyes (propidium iodide and SYTO9 fluorescent stain) were used to double stain the removed titanium K-wires. Place the prepared K-wires under CLSM to observe the distribution of bacteria on the surface of the K-wires.

\section{Statistical analysis}

Statistical analysis was performed using SPSS22.0 statistical software. Measurement data are expressed as mean \pm standard deviation $(x \pm s)$, using t test. A p-value $<0.05$ was considered statistically significant.

\section{Ethics statement.}

The study protocol was approved by the Experimental Animal Ethics Committee of 900th Hospital of PLA. All methods were carried out in accordance with relevant guidelines and regulations.

\section{Results}

On the third day after the operation, one rabbit in the non-iodine-coated group died. A specimen of the affected limb was taken and sent for microbiological culture and pathological examination. The results were not included in the statistical data. the other rabbits survived one week after surgery.

\section{Macroscopic score of wounds}


In the iodine-coated group, the injured limb wounds of rabbits were generally in good condition, and the mean gross wound score was $0.5 \pm 0.76$ points(as shown in Fig. 1 - g). In the non-iodine-coated group, signs of infection were found in the wounds of rabbits, and the gross wound score was $1.57 \pm 0.54$ points(as shown in Fig. 1 - $f$ ). The gross wound score of the iodine-coated group was lower than that of the non-iodine-coated group(as shown in Table 1), the basic information of the experimental rabbits can be found in Supplementary Table S1 online, with $P$ value of $0.008(P<0.05)$, and the difference was statistically significant.

Table 1

Wound gross observation score statistical table

\begin{tabular}{|llll|}
\hline & The iodine-coated group & The non-iodine-coated group & $P$-value \\
\hline Mean score & $0.5 \pm 0.76$ & $1.57 \pm 0.54$ & 0.008 \\
\hline
\end{tabular}

\section{Microbiological evaluation}

Each experimental animal is sent for 2 specimens. Of the 7 cases in the iodine-coated group, 5 of the 7 cases were positive for bacterial culture, and 2 cases were negative, with a positive rate of $71.4 \%$.Of the 8 cases in the iodine-coated group, 3 cases were positive for culture and 5 cases were negative for culture, with a positive rate of 37.5 \%(as shown in Fig. 2).It was identified as Escherichia coli by an automatic microorganism identification instrument. The rabbit that died on the third day after the operation had a positive bacterial culture and was also identified as Escherichia coli by an automatic microbial identification instrument.

\section{Histopathologic evaluation}

All bone tissue specimens showed different degrees of osteomyelitis under the light microscope.The statistical results of the diagnosis score are shown in Table 2. Detailed statistics for the jupiter score can be found in Supplementary Table S2 online. The score of the iodine-coated group is $9.75 \pm 1.67$ points, and the score of the non-iodine-coated group is $11.43 \pm 1.81$ points. The results showed that the two groups of specimens met a major acute diagnostic criteria ${ }^{26}$ and the score was $\geq 6$ points. The results indicated that acute osteomyelitis could be diagnosed in both groups. The severity of infection score is shown in Table 3. Raw pathological score data can be found in Supplementary Table S3 online. The pathology score of the non-iodine-coated iodine group is $20.29 \pm 2.14$ points, and the iodine-coated group is $13.75 \pm 2.49$ points $(P<0.001)$, the results show The difference in histopathological score between the two groups was statistically significant. The severity of infection in the iodine-coated group was significantly lower than that of the non-iodine-coated group. Among them, the intramedullary score was $7.5 \pm 1.41$ points in the iodine-coated group and $11.71 \pm 1.38$ points in the non-iodine-coated group ( $\mathrm{P} \otimes 0.001$ ), and the difference was statistically significant. The score of the cortical bone part was $4.75 \pm 1.04$ points for the iodine-coated group and $6.57 \pm 1.51$ points for the non-iodine-coated group $(P<0.05)$, and the difference was statistically significant. The periosteal reaction score was $1.25 \pm 1.04$ points for the iodine-coated group and $1.71 \pm 0.76$ points for the non-iodine-coated group $(P>0.05)$, and the difference was not statistically significant. In the bone tissue specimens of the rabbits that died on the third day after the operation, there were a large number of neutrophils in the medullary cavity. Each high-powered field of view is $\geq 10$ neutrophils. Jupiter score $>6$ points, consider the presence of acute osteomyelitis.

Table 2 Jupiter score statistics 


\begin{tabular}{|lll|}
\hline & The iodine-coated group & The non-iodine-coated group \\
\hline Total score & $9.75 \pm 1.67$ & $11.43 \pm 1.81$ \\
\hline
\end{tabular}

Table 3 Histopathological severity score results

\begin{tabular}{|c|c|c|c|}
\hline & The iodine-coated group & The non-iodine-coated group & $P$-value \\
\hline Intramedullary cavity & $7.50 \pm 1.41$ & $11.71 \pm 1.38$ & $\llbracket 0.001$ \\
\hline Cortical bone & $4.75 \pm 1.04$ & $6.57 \pm 1.51$ & 0.016 \\
\hline Periosteal reaction & $1.25 \pm 1.04$ & $1.71 \pm 0.76$ & 0.336 \\
\hline total score & $13.75 \pm 2.49$ & $20.29 \pm 2.14$ & $\otimes 0.001$ \\
\hline
\end{tabular}

Table 4 Statistical table of histopathological observations

\begin{tabular}{|lllllllll|}
\hline \multicolumn{2}{|c|}{ Medullary canal } & \multicolumn{2}{c}{ Cortex } & & Periosteum \\
\hline $\begin{array}{l}\text { Neutrop- } \\
\text { hils }\end{array}$ & $\begin{array}{l}\text { Microab- } \\
\text { scess }\end{array}$ & $\begin{array}{l}\text { Granulation } \\
\text { tissue }\end{array}$ & $\begin{array}{l}\text { Fibros- } \\
\text { is }\end{array}$ & $\begin{array}{l}\text { Destructio- } \\
\text { n of cortex }\end{array}$ & $\begin{array}{l}\text { Enlarged } \\
\text { Haversiaii } \\
\text { canals }\end{array}$ & $\begin{array}{l}\text { Fibros- } \\
\text { is }\end{array}$ & $\begin{array}{l}\text { Periosteal } \\
\text { reaction }\end{array}$ \\
\hline $\begin{array}{l}\text { The } \\
\text { iodine- } \\
\text { coated } \\
\text { group }\end{array}$ & 8 & 8 & 3 & 2 & 8 & 8 & 0 & 5 \\
$\begin{array}{l}\text { The } \\
\text { non- } \\
\text { iodine- } \\
\text { coated } \\
\text { group }\end{array}$ & 7 & 7 & 7 & 3 & 7 & 7 & 1 & 6 \\
\hline
\end{tabular}

\section{SEM observation results}

Figures $a$ and $b$ are respectively the results of scanning the surface of the titanium $\mathrm{K}$-wires without iodine and the surface of the titanium K-wires with iodine under a 5000x microscope(as shown in Fig. 4). From Figure a, we can see that there are horizontal protrusions of different depths on the surface of the non-iodine-coated K-wires, and Escherichia coli is densely attached to the surface of the K-wires. E. coli can be observed on the surface of non-iodine-coated titanium K-wires in 5 samples submitted for inspection, of which 3 samples can be observed densely distributed on the surface of non-iodine-coated K-wires, and 2 samples can be observed scattered and attached to the K-wires.Figure $A$ is representative.It can be seen from Figure $b$ that the surface of the iodinecoated K-wire is covered with a dense and uniform coating. On the surface of the coating, there are scattered shallow strips of different sizes. Escherichia coli is only occasionally visible on the surface of the coating(As shown in Figure b). E. coli was occasionally observed on the surface of the iodine-coated K-wires in 4 of the 7 submitted samples, while no E. coli was observed on the surface of iodine-coated K-wires in the remaining 3 samples.

\section{CLSM observation results}


The non-iodine-coated group sent 6 specimens, and The iodine-coated group sent 5 specimens.It can be seen from the figure that live bacteria turn green in fluorescence, and dead bacteria turn red (Fig. 5). Figure a is the scan results of the K-wires without iodine. In 6 specimens, a large number of densely distributed green fluorescence and a small amount of red fluorescence on the surface of the non-iodine-coated K-wires can be observed. The green fluorescence distribution is densely clustered with clusters. A small part is distributed in dots, and the red fluorescence is scattered in dots. Figure b is the scan result of the iodine-coated K-wire. From 5 specimens of the iodine-coated K-wires, it can be observed that the green fluorescence and red fluorescence on the surface of the iodine-coated K-wires are scattered in dots, and the fluorescence distribution is relatively sparse.

\section{Discussion}

Currently, the concept of preventing implant infection emphasizes the importance of implant surface and surrounding tissues ${ }^{17}$.Due to its clear antibacterial effect and rare occurrence of pathogen resistance, PVP-I has been paid close attention and further studied by Professor Shirai et al. ${ }^{18-21}$.The iodine-loaded implants prepared by our team have been confirmed to have exact antibacterial properties against Staphylococcus aureus through in vivo and in vitro antibacterial experiments ${ }^{21-23}$.

In this study, our results showed that the iodine-coated titanium K-wires had a clear antibacterial effect on Escherichia coli in the early stage of infection. It can be inferred from the gross wound score and microbiological culture results of the two groups that the severity of wound infection in the iodine-loaded group was lower than that in the unloaded group. Histopathological observations confirmed this inference. Combining the statistical results in Table 4 and Fig. 3, there were 5 typical histomathological changes of E. coli associated acute osteomyelitis: 1. A large number of neutrophils ( $\geq 10$ neutrophils / 1HPF) could be seen in the medullary cavity clustered in the space between adipocytes; 2 . Focal neutrophil aggregation (i.e. microabscess) is seen in the medullary cavity; 3 . Local bone erosion and bone destruction were observed in in the cortical bone; 4 . Harvard system enlargement can be seen in the cortical bone; 5 . Local periosteum reactions are common. This is roughly consistent with the description of the histopathological manifestations of acute osteomyelitis in the previous literature and experimental studies, focal neutrophil aggregation can be seen in the medullary cavity ${ }^{29,30}$,the most common signs of cortical bone infection were periosteum thickening, enlargement of Haver's canal, and destruction of the cortex. Only a few specimens showed leukocytes, fibrosis and granulation tissue, and no microabscesses were found ${ }^{31}$.There are two possible reasons for the difference between the observation results of SEM and CLSM: First, the sensitivity and specificity of the two observation methods are different, SEM has high specificity and poor sensitivity, while CLSM has specificity Inferior and high sensitivity;secondly, the sample processing procedure before SEM observation is more and more complicated than that of CLSM, and the bacteria on the SEM sample may partly fall off during the processing.

The improved animal model in this study has the following three advantages: First, the total amount of E. coli inoculated in this experiment is $5 \times 10^{6} \mathrm{CFU}$, and the bacterial fluid volume is lower; second, the experimental animal model of osteomyelitis in this study has lower mortality ( The mortality rate is 6.25\%); Finally, in this study, $5 \%$ sodium morrhuate was not added as a sclerosing agent to assist in the construction of animal models during the experiment. 
Of course, this study also had several limitations: 1 . This experiment only studies the short-term antibacterial performance of iodine-coated K-wires against Escherichia coli, and the long-term antibacterial performance of antibacterial coatings is also very important. In the future, it can be carried out. The sustained-release experimental study of the wire in animals was used to verify the antibacterial performance of the iodine-loaded titanium K-wires after long-term implantation in the animal body. 2. The safety performance measurement and evaluation of iodine-coated K-wires need to be further studied through animal experiments in the future.

\section{Conclusions}

The results of this experiment showed that the iodine-coated titanium implant could effectively inhibit the infection of Escherichia coli in the early stage of infection. Therefore, we believe that the iodine-coated titanium implant prepared by our team has high research value and application prospect in the field of clinical orthopedics.

\section{Declarations}

\section{Data availability}

All data needed to evaluate the conclusions in the paper are present in the paper and the Supplementary Information materials.

\section{Acknowledgements}

We wish to thanks Mr. Wang, who was a Professor of Fujian Medical University, for considerable advice on experimental design and interpretation of results. We also thank Qiping Wu and Hao Zeng for their assistance during the experiment.

\section{Author contributions}

X.Z. wrote the manuscript. X. Z.,W.Y. and Y.Z. conducted the experiments.X.Z. and W.W. involved in the acquisition, analysis and interpretation of experimental data. X.Z. and W.W. designed the experimental study.

\section{Competing interests}

The authors declare no competing interests.

Ethics approval This study was performed with the approval of the animal ethics committee at our hospital (Approval date: September 20, 2020).

\section{References}

1. Eliaz Noam. Corrosion of Metallic Biomaterials: A Review.Materials (Basel), 12(3). ( 2019).

2. Del Pozo Jose, L. Patel Robin.Clinical practice. Infection associated with prosthetic joints. N Engl J Med. 361 (8), 787-794 (2009).

3. Harris Llinos, G., Richards, R. \& Geoff Staphylococci and implant surfaces: a review. Injury, null(undefined), S3-14(2006). 
4. Zimmerli, W. Clinical presentation and treatment of orthopaedic implant-associated infection. J Intern Med. 276 (2), 111-119 (2014).

5. Bouvet, C. et al. No need to search for the source of haematogenous arthroplasty infections. Swiss Med Wkly. 141 (undefined), w13306 (2011).

6. Rodríguez-Pardo, D. et al. REIPI Group for the Study of Prosthetic Infection.Gram-negative prosthetic joint infection: outcome of a debridement, antibiotics and implant retention approach. A large multicentre study. Clin Microbiol Infect. 20 (11), 0911-9 (2014).

7. McConoughey, S. J. et al. Biofilms in periprosthetic orthopedic infections. Future Microbiol. 9 (8), 987-1007 (2014).

8. Schmidmaier, G., Lucke, M., Wildemann, B., Haas, N. P. \& Raschke, M. Prophylaxis and treatment of implantrelated infections by antibiotic-coated implants: a review. Injury. 37 (Suppl 2), S105-S112 (2006).

9. Kim, W. H. et al. The release behavior of chx from polymer-coated titanium surfaces. Surface \& Interface Analysis. 40 (3-4), 202-204 (2008).

10. Mijnendonckx, K., Leys, N., Mahillon, J., Silver, S. \& Van Houdt, R. Antimicrobial silver: uses, toxicity and potential for resistance. Biometals. 26 (4), 609-621 (2013).

11. Caro, A. et al. Grafting of lysozyme and/or poly(ethylene glycol) to prevent biofilm growth on stainless steel surfaces. J Phys Chem B. 113 (7), 2101-2109 (2009). )

12. Peng, Z. et al. Dual effects and mechanism of TiO2 nanotube arrays in reducing bacterial colonization and enhancing С3H10T1/2 cell adhesion. Int J Nanomedicine, 8(undefined), 3093 - 105(2013).

13. Li, T. et al. Antibacterial activity and cytocompatibility of an implant coating consisting of TiO nanotubes combined with a GL13K antimicrobial peptide. Int J Nanomedicine. 12 (undefined), 2995-3007 (2017).

14. Tan, H., Ma, R., Lin, C., Liu, Z. \& Tang, T. Quaternized chitosan as an antimicrobial agent: antimicrobial activity, mechanism of action and biomedical applications in orthopedics. Int J Mol Sci. 14 (1), 1854-1869 (2013).

15. Martinez-Pastor, J. C. et al. Antibiotic resistance in orthopaedic surgery: acute knee prosthetic joint infections due to extended-spectrum beta-lactamase (ESBL)-producing Enterobacteriaceae. Eur J Clin Microbiol Infect Dis. 29 (8), 1039-1041 (2010).

16. Pountos, I. et al. Mesenchymal Stem Cell physiology can be affected by antibiotics: An in vitro study. Cell Mol Biol (Noisy-le-grand). 60 (4), 1-7 (2014).

17. Alt, V. Antimicrobial coated implants in trauma and orthopaedics-A clinical review and risk-benefit analysis. Injury. 48 (3), 599-607 (2017).

18. Shirai, T. et al. Antibacterial iodine-supported titanium implants. Acta Biomater. 7 (4), 1928-1933 (2011).

19. Tsuchiya, H. et al. Innovative antimicrobial coating of titanium implants with iodine. J Orthop Sci. 17 (5), 595-604 (2012).

20. Kato, T. \& Shirai, T. Temporal attenuation of iodine content and its effect on the antibacterial activity of iodine-supported titanium implants. Journal of Microbial \& Biochemical Technology, 8(4).(2016).

21. Inoue, D. et al. Inhibition of biofilm formation on iodine-supported titanium implants. Int Orthop. 41 (6), 1093-1099 (2017).

22. Yang, X. et al. Preparation of the iodine $\nabla$ coating titanium implant in orthopedics and evaluation of antibacterial activity. Chinese Journal of Orthopaedics. 38 (22), 1366-1373 (2018). 
23. Wan, B. et al. A Study on Fabrication of the lodine-coating Titanium Platewith Potassium lodide and Its Antibacterial Property. Chinese Journal of Traumatology and Orthopedics. 22 (3), 249-254 (2020).

24. Xie, Z. et al. Gentamicin-loaded borate bioactive glass eradicates osteomyelitis due to Escherichia coli in a rabbit model. Antimicrob Agents Chemother. 57 (7), 3293-3298 (2013).

25. Tang, H. \& Xu, Y. Relationship between inoculation dose and rabbit model of staphlococcus osteomyeliti s. Orthopedic Journal of China. (2009).

26. Sybenga, A. B. et al. Diagnosing Osteomyelitis: A Histology Guide for Pathologists. J. Foot Ankle Surg. 59 (1), 75-85 (2020).

27. Smeltzer, M. S. et al. Characterization of a rabbit model of staphylococcal osteomyelitis. J Orthop Res. 15 (3), 414-421 (1997).

28. Vogely, H. C. et al. Effects of hydrosyapatite coating on Ti-6A1-4V implant-site infection in a rabbit tibial model. J Orthop Res. 18 (3), 485-493 (2000).

29. Tiemann, A. et al. Histopathological Osteomyelitis Evaluation Score (HOES) - an innovative approach to histopathological diagnostics and scoring of osteomyelitis. GMS Interdiscip Plast Reconstr Surg DGPW, 3(undefined), Doc08(2014).

30. Schmidt, H. G. K. et al. Arbeitsgemeinschaft septische Chirugie der DGOUC10. [Definition of the Diagnosis Osteomyelitis-Osteomyelitis Diagnosis Score (ODS)]. Z Orthop Unfall. 149 (4), 449-460 (2011).

31. Moojen, D. J. F. et al. No efficacy of silver bone cement in the prevention of methicillin-sensitive Staphylococ -cal infections in a rabbit contaminated implant bed model. J Orthop Res. 27 (8), 1002-1007 (2009).

\section{Figures}

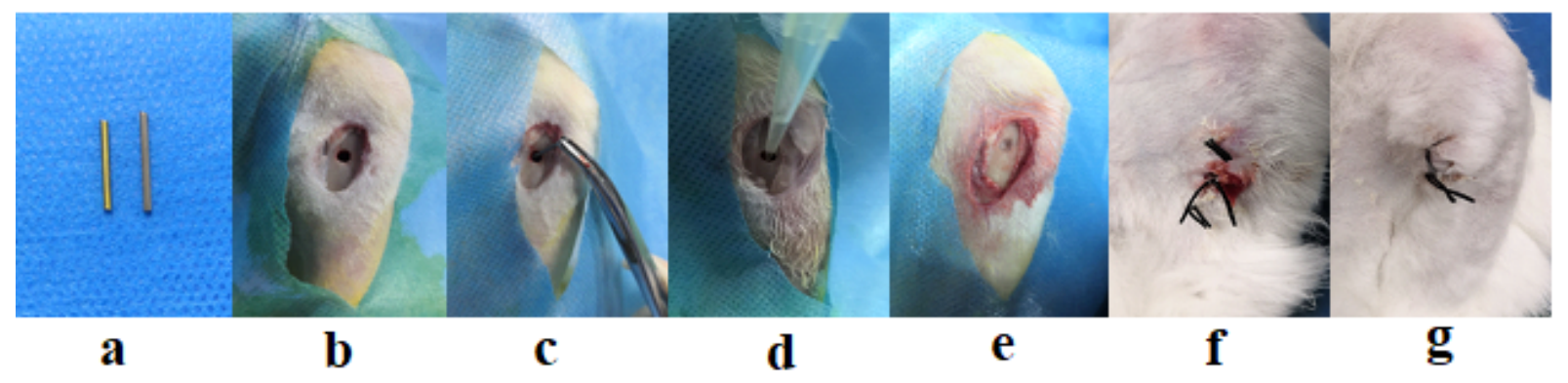

Figure 1

Experimental process and general appearance of the wound.a non-iodine-coated titanium K-wires and iodinecoated titanium K-wires.b-e The experimental process.b Drill the proximal end of the rabbit's left tibia.c Implantation of K-wirs in the medullary cavity of rabbit tibia.d Bacterial suspension was injected into the bone marrow cavity of the tibia of rabbits.e Bone wax to close the bone window. $f$ The wounds of the affected limbs of the rabbits in the non-iodine -coated group showed purulent exudate in the wounds one week after the operation.g The wound condition of the affected limb of the rabbits in the iodine-loaded group one week after the operation, it can be seen that the wound is in good condition without purulent exudate; 

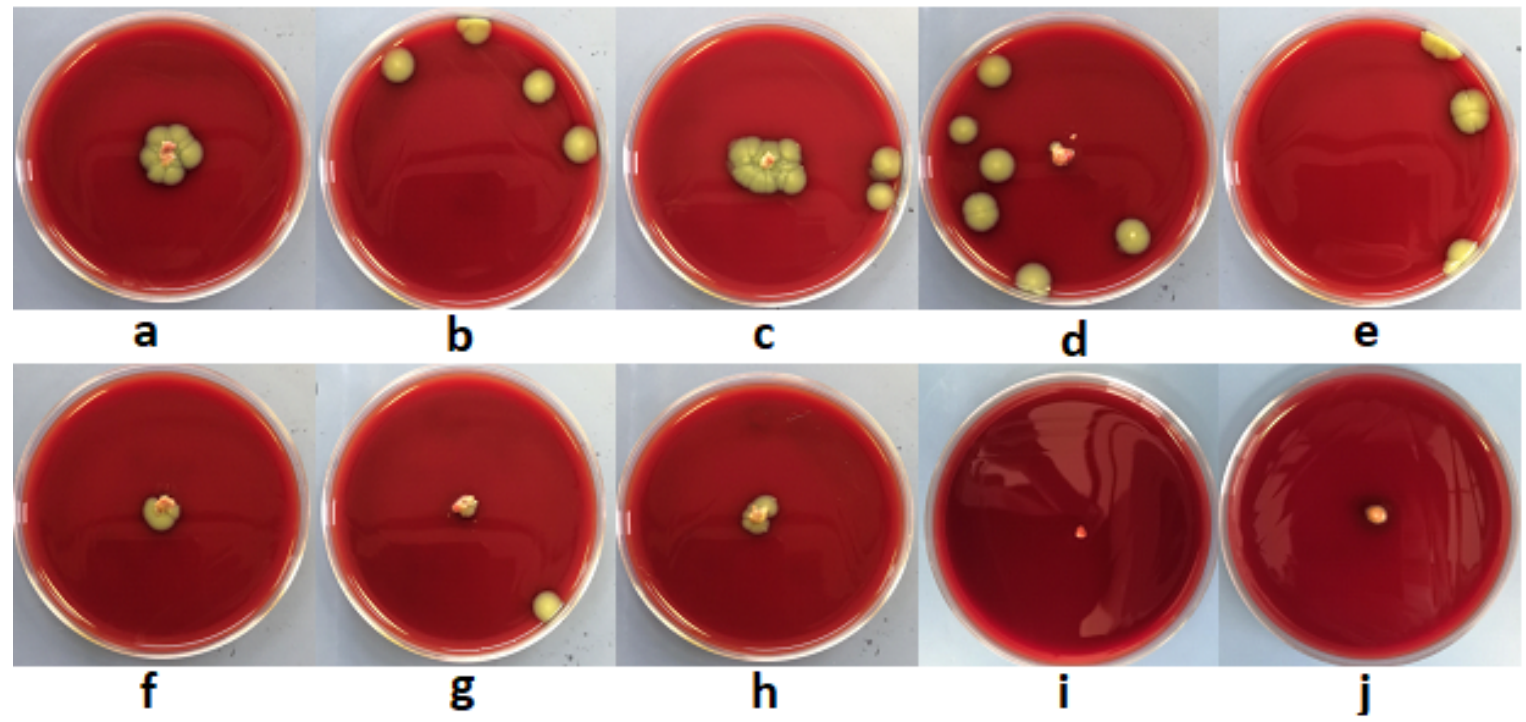

Figure 2

a-e Bacterial culture results of the non-iodine-coated group, $\mathrm{f}-\mathrm{j}$ Bacterial culture results of The iodine-coated group. 

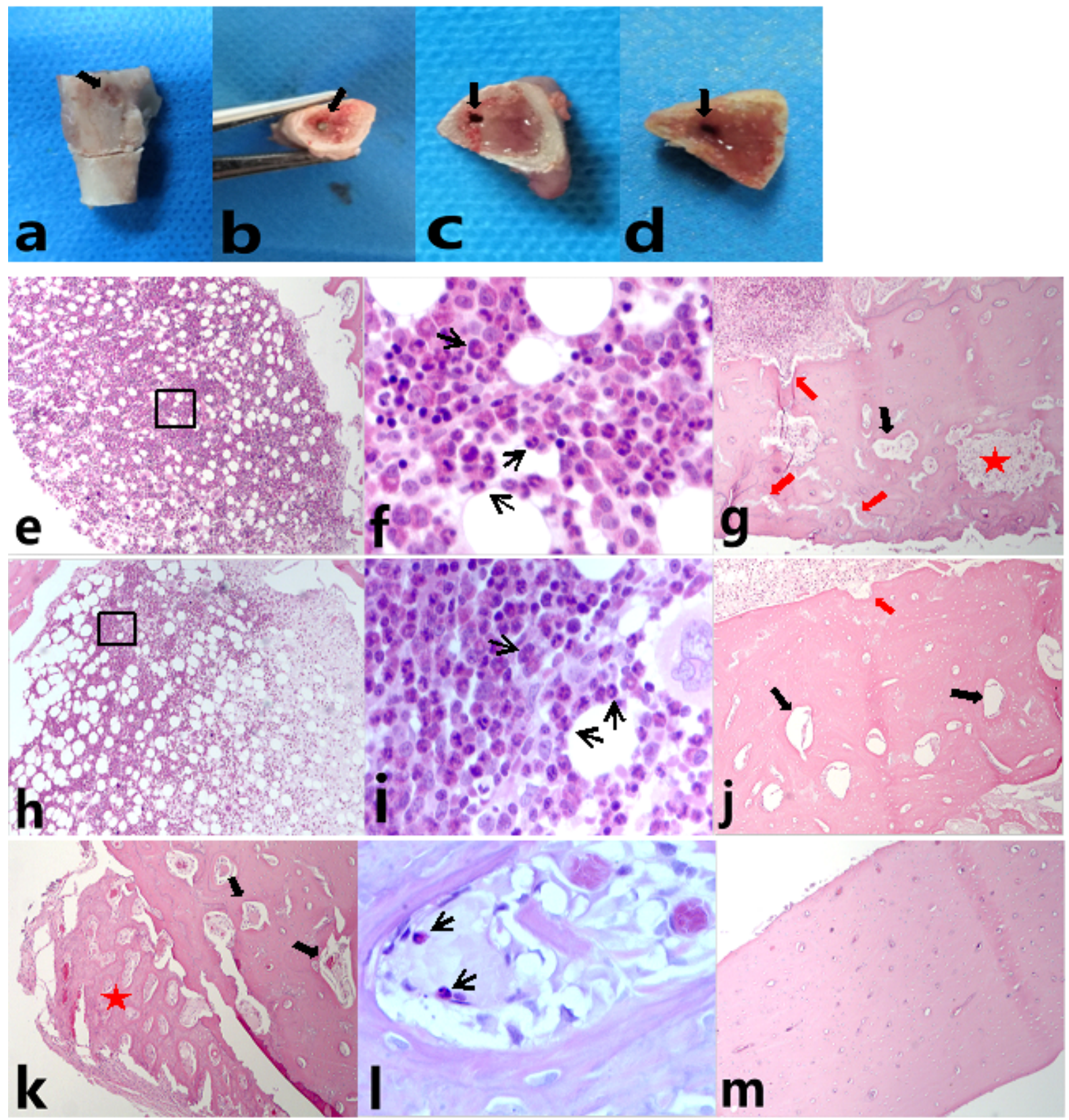

Figure 3

a-d Pathological specimens from the experiment. e-m The pathological specimen stained with HE under microscope. a Histopathological bone specimen, shown by the black arrow, is the tibial borehole. b K-wires implanted in the medullary cavity. c The location of the Kirschner pin track (shown by the black arrow). e-g The performance of the intramedullary cavity and cortical bone under light microscope in the non-iodine-coated group.Figure $f$ is the box in Figure e under a 400x magnification.A large number of neutrophils (shown by the black arrow in the figure $f$ ) can be seen under the light microscope. In the cortex, enlargement of the Harvard system (shown by the black arrow in the figure g), bone erosion and bone destruction (shown by the red arrow in the figure $\mathrm{g}$ ), and fibrotic changes (shown by the red star in the figure $\mathrm{g}$ ) can be seen in the cortex. h-j Light microscopy and intramedullary and cortical appearance of iodine-coated group.Figure i shows the box in figure $h$ under a 400x magnification, A large number of neutrogranulocytes (shown by the black arrow in figure i) can also be seen in the medullary cavity under light microscopy. The area of microabscess is smaller than that in the 
uniodide group. An enlarged Harvard's system can be seen in the bone cortex (shown by the black arrow in figure j). $k$ Local periosteum reaction,the reaction subperiosteal bone formation visible (shown with red stars ), seen in figure enlarged Haversian systems (shown with black arrows). I Occasionally sees neutrophils in cortical bone

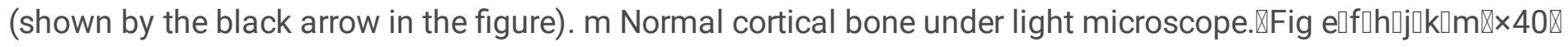

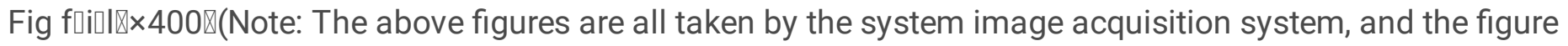
display range is only about $1 / 6$ of the range seen under the lens.)

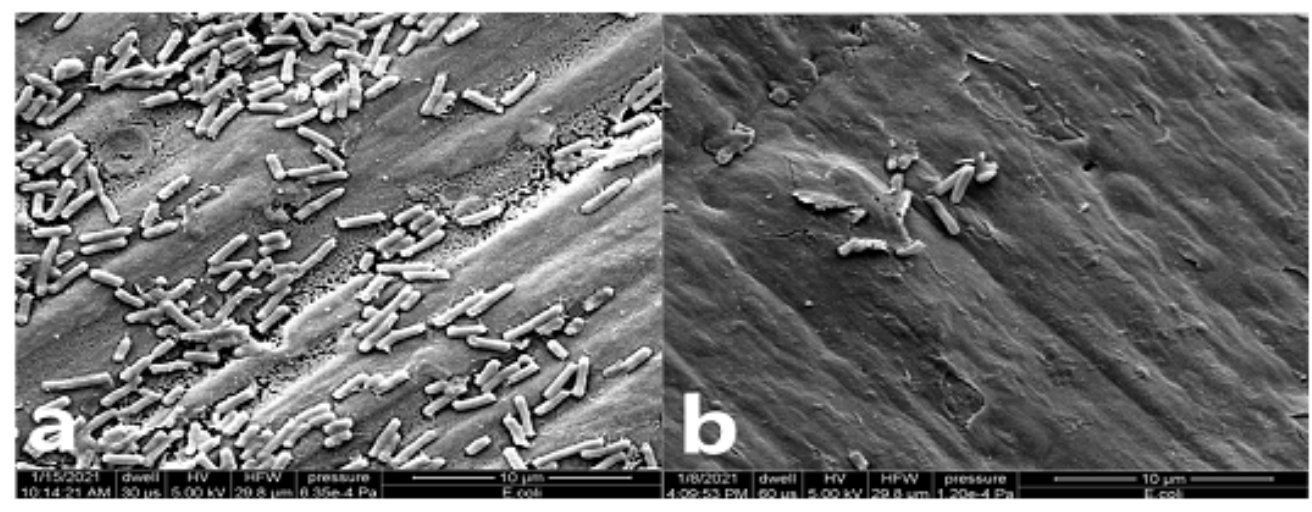

\section{Figure 4}

Scanning electron microscopy observation results of non-iodine-coated K-wires and iodine-coated K-wires. a The surface of the K-wire without iodine-coated group (5000X); b The surface of the K-wire with iodine-coated group (5000X).

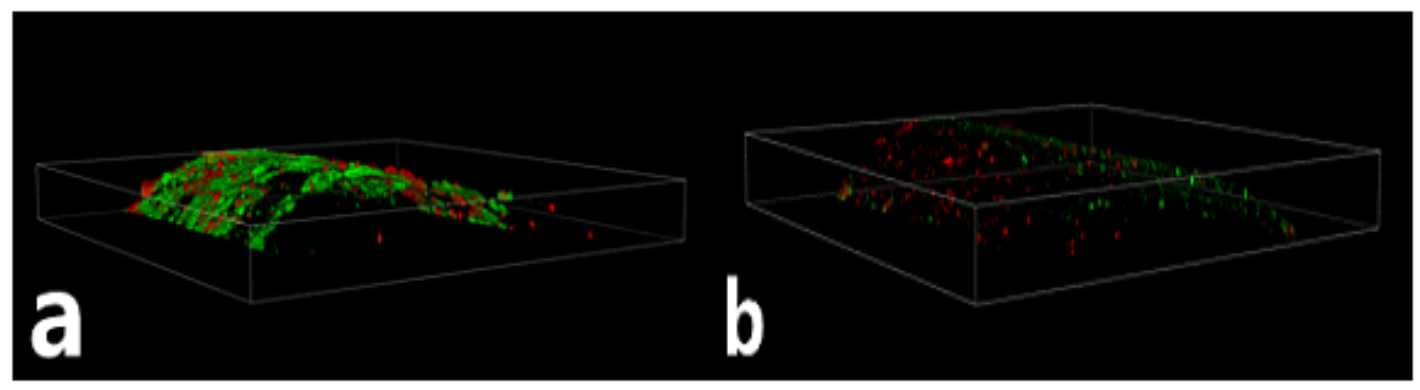

\section{Figure 5}

Scanning results of CLSM, a the scanning results of the non-iodine-coated group. $b$ the scanning results of The iodine-coated group. 